\title{
Pacific
}

Journal of

Mathematics

\section{DUALITY FOR THE QUANTUM $E(2)$ GROUP}

Alfons Van Daele and S. L. Woronowicz 


\section{DUALITY FOR THE QUANTUM E(2) GROUP}

\section{A. Van Daele and S.L. Woronowicz}

The quantum deformation $E_{\mu}(2)$ of the group of motions of the plane on the $\mathrm{C}^{*}$-level was obtained by the second author. He also constructed the Pontryagin dual $\widehat{E}_{\mu}(2)$ explicitly, together with the unitary bicharacter describing this duality. In this paper we show that the dual of $\widehat{E}_{\mu}(2)$ is again $E_{\mu}(2)$.

\section{Introduction.}

Let $\mu$ be any number in $] 0,1\left[\right.$. Consider the ${ }^{*}$-algebra $A$ over $\mathbb{C}$ with an identity, generated by a unitary element $v$ and a normal element $n$ satisfying $v n=\mu n v$. This ${ }^{*}$-algebra can be made into a Hopf ${ }^{*}$-algebra when the comultiplication $\Phi$ is defined by

$$
\begin{aligned}
& \Phi(v)=v \otimes v \\
& \Phi(n)=v \otimes n+n \otimes v^{*} .
\end{aligned}
$$

Then, the counit $\epsilon$ is given by $\epsilon(v)=1$ and $\epsilon(n)=0$. The antipode $S$ is given by $S(v)=v^{*}$ and $S(n)=-\mu^{-1} n$.

On the other hand, consider the *-algebra $B$ over $\mathbb{C}$ with identity, generated by a self-adjoint invertible element $a$ and a normal element $b$ satisfying $a b=\mu b a$. Again $B$ can be made into a Hopf *-algebra when $\Phi$ is defined by

$$
\begin{aligned}
& \Phi(a)=a \otimes a \\
& \Phi(b)=a \otimes b+b \otimes a^{-1} .
\end{aligned}
$$

Then, the counit is given by $\epsilon(a)=1$ and $\epsilon(b)=0$ while the antipode is determined by $S(a)=a^{-1}$ and $S(b)=-\mu^{-1} b$.

The pair $(A, B)$ becomes a dual pair of Hopf *-algebras when the pairing is defined by

$$
\begin{array}{llrl}
\langle v, a\rangle & =\mu^{\frac{1}{2}} & & \langle n, a\rangle=0 \\
\langle v, b\rangle=0 & & \langle n, b\rangle=1 .
\end{array}
$$

This pairing is non-degenerate. (For details, see e.g. $[5,6]$ and $[8]$.) 
The algebra $A$ can be considered as a quantized version - on the Hopf *-algebra level - of the group $E(2)$ of motions of the plane. To obtain these two quantum groups on the $\mathrm{C}^{*}$-algebra level (i.e. the quantized version of the continuous functions on the group that tend to zero at infinity), turns out to be non-trivial and certainly more complicated than for the examples of compact quantum groups and the (non-compact) Heisenberg group (see e.g. [9]). The reason is the following. When representing the algebras $A$ and $B$ by means of operators on a Hilbert space $\mathcal{H}$, it turns out that, although the elements $\Phi(n)$ and $\Phi(b)$ are normal in the *algebras $A \otimes A$ and $B \otimes B$ respectively, these elements are only formally normal as operators on $\mathcal{H} \otimes \mathcal{H}$. In fact, only with certain restrictions on the spectrum, these operators have proper normal extensions. This is necessary to define the comultiplication on the $\mathrm{C}^{*}$-level. (See [1] and [7].)

It was the second author who developed the necessary machinery to deal with these problems. In [1] the theory of unbounded elements affiliated with a $\mathrm{C}^{*}$-algebra is developed and this is used to obtain the quantization $E_{\mu}(2)$ of the group of motions of the plane on the $\mathrm{C}^{*}$-level. In [3] the results on pairs of normal operators satisfying certain commutation rules are obtained. And in [2] this is used to obtain the dual $\widehat{E}_{\mu}(2)$. It turns out that this is indeed the $\mathrm{C}^{*}$-version of the second Hopf ${ }^{*}$-algebra described above.

In this paper, we use the same techniques as in [2] to show that the Pontryagin dual of $\widehat{E}_{\mu}(2)$ is again $E_{\mu}(2)$. This is done in section 2 of the paper. In section 1 we recall the definitions of $E_{\mu}(2)$ and $\widehat{E}_{\mu}(2)$ and we collect some of the properties that we need on $\widehat{E}_{\mu}(2)$ and its automorphisms.

\section{The quantum groups $E_{\mu}(2)$ and $\widehat{E}_{\mu}(2)$.}

Fix a number $\mu \in] 0,1\left[\right.$. The quantum group $E_{\mu}(2)$ on the $\mathrm{C}^{*}$-algebra level can be described as a $\mathrm{C}^{*}$-crossed product in the following way. Denote

$$
\overline{\mathbb{C}}^{\mu}=\left\{z \in \mathbb{C} \mid z=0 \quad \text { or } \quad|z| \in \mu^{\mathbb{Z}}\right\} .
$$

Let $C_{\infty}\left(\overline{\mathbb{C}}^{\mu}\right)$ be the algebra of continuous complex functions on $\overline{\mathbb{C}}^{\mu}$, vanishing at infinity. Define an automorphism $\alpha$ by $(\alpha f)(z)=f(\mu z)$. Then the $\mathrm{C}^{*}$-algebra $A$ is defined as the crossed product $C_{\infty}\left(\overline{\mathbb{C}}^{\mu}\right) \times_{\alpha} \mathbb{Z}$.

Given a Hilbert space $\mathcal{H}$, a unitary operator $v$ and a normal operàtor $n$ with $\operatorname{Sp}(n) \subseteq \overline{\mathbb{C}}^{\mu}$ such that $v n v^{*}=\mu n$, there exists a covariant representation of the above covariant system given by $\pi(f)=f(n)$ since $v \pi(f) v^{*}=f\left(v n v^{*}\right)=f(\mu n)=(\alpha f)(n)$. Hence there is an associated representation of $A$ on $\mathcal{H}$. 
If we take $\mathcal{H}=\ell^{2}(\mathbb{Z} \times \mathbb{Z})$ and we define $v$ and $n$ by

$$
\begin{aligned}
(v \xi)(k, \ell) & =\xi(k+1, \ell) \\
(n \xi)(k, \ell) & =\mu^{k} \xi(k, \ell-1),
\end{aligned}
$$

then the pair satisfies the above requirements. In fact, we have sucb universal pair in the sense that the corresponding representation of $A$ faithful. This means that $A$ can be identified with the $\mathrm{C}^{*}$-algebra obtained by taking the closed linear span of operators of the form $v^{k} f(n)$ where $k \in \mathbb{Z}$ and with $f \in C_{\infty}\left(\overline{\mathbb{C}}^{\mu}\right)$.

If we take the pair $(v, n)$ and we define

$$
\begin{aligned}
& \widetilde{v}=v \otimes v \\
& \widetilde{n}=v \otimes n+n \otimes v^{*}
\end{aligned}
$$

(by $\dot{+}$ we mean the closure of the sum of the two operators), it follows from the results in $[\mathbf{3}]$ that we get again a pair with the same requirements. Hence there is a *-representation $\Phi$ of $A$ induced by this pair. This $\Phi$ is a morphism of $A$ into $M(A \otimes A)$ and it is coassociative.

Definition 1.1. The quantum group $E_{\mu}(2)$ is the $\mathrm{C}^{*}$-algebra $A$ together with this comultiplication.

The quantum group $\widehat{E}_{\mu}(2)$ is a little more difficult to describe.

Denote by $\bar{\Sigma}_{\mu}$ the closure of the set

$$
\Sigma_{\mu}=\left\{\left(s, \mu^{r}\right) \mid s \in \mathbb{Z}, r \in \mathbb{Z}+\frac{s}{2}\right\}
$$

and consider again the $\mathrm{C}^{*}$-algebra $C_{\infty}\left(\bar{\Sigma}_{\mu}\right)$. Define an automorphism $\beta$ on this $\mathrm{C}^{*}$-algebra by $(\beta f)\left(s, \mu^{r}\right)=f\left(s-2, \mu^{r}\right)$. Let $B_{1}$ be the $\mathrm{C}^{*}$-crossed product $C_{\infty}\left(\bar{\Sigma}_{\mu}\right) \times_{\beta} \mathbb{Z}$. We will consider the $\mathrm{C}^{*}$-subalgebra $B$ of $B_{1}$ generated by finitely supported functions $\xi: \mathbb{Z} \rightarrow C_{\infty}\left(\bar{\Sigma}_{\mu}\right)$ such that $(\xi(k))(r, 0)=0$ for all $r$ when $k \neq 0$.

Given a Hilbert space $\mathcal{H}$, a self-adjoint operator $N$ and a normal operator $b$ with polar decomposition $b=u|b|$ such that $N$ and $|b|$ strongly commute and have joint spectrum $\operatorname{Sp}(N,|b|) \subseteq \bar{\Sigma}_{\mu}$ and such that $u$ is a unitary and satisfies $u N u^{*}=N-2$, there is a covariant representation of the above covariant system given by $\pi(f)=f(N,|b|)$ since $u \pi(f) u^{*}=f(N-2,|b|)=$ $(\beta f)(N,|b|)$. Then we also get an associated representation of the $\mathrm{C}^{*}$-algebra $B_{1}$. If $u$ is no longer unitary, but only a partial isometry satisfying $u N=$ 
$(N-2) u$, we still have $u \pi(f)=(\beta f)(N,|b|) u$ and by the choice of $B$ there is still an associated representation of the $C^{*}$-algebra $B$.

Take $\mathcal{H}=\ell^{2}\left(\Sigma_{\mu}\right)$ and define $N$ and $b$ by

$$
\begin{aligned}
(N \xi)\left(s, \mu^{r}\right) & =s \xi\left(s, \mu^{r}\right) \\
(b \xi)\left(s, \mu^{r}\right) & =\mu^{r} \xi\left(s-2, \mu^{r}\right) .
\end{aligned}
$$

Then we have operators as above and the pair is universal in the sense that the corresponding representation of $B_{1}$ is faithful. We again identify $B_{1}$ with the $\mathrm{C}^{*}$-algebra obtained by taking the closed linear span of operators of the form $u^{k} f(N,|b|)$ where $k \in \mathbb{Z}$ and $f \in C_{\infty}\left(\bar{\Sigma}_{\mu}\right)$. Then $B$ is the closed linear span of the operators $u^{k} f(N,|b|)$ with $f(r, 0)=0$ if $k \neq 0$.

The comultiplication on $B$ is defined as follows. Consider the universal pair $(N, b)$ as above. Let

$$
\begin{aligned}
\tilde{N} & =N \otimes 1+1 \otimes N \\
\tilde{b} & =b \otimes \mu^{\frac{1}{2} N} \dot{+} \mu^{-\frac{1}{2} N} \otimes b
\end{aligned}
$$

where $\mu^{\frac{1}{2} N}$ is $f(N)$ with $f(k)=\mu^{\frac{1}{2} k}$ for $k \in \mathbb{Z}$ and similarly for $\mu^{-\frac{1}{2} N}$. It follows from [3] that this pair again satisfies the right properties and hence defines a representation. This gives the comultiplication $\Phi: B \rightarrow M(B \otimes B)$. Definition 1.2. The quantum group $\widehat{E}_{\mu}(2)$ is the $C^{*}$-algebra $B$ together with this comultiplication.

In [2] it is shown that $\widehat{E}_{\mu}(2)$ is the Pontryagin dual of $E_{\mu}(2)$. We will show that also the dual of $\widehat{E}_{\mu}(2)$ is again $E_{\mu}(2)$. The method that we use is very similar to the one used in [2].

First we want to obtain some more results on the quantum group $\widehat{E}_{\mu}(2)$.

Define a projection operator $Q$ on $\ell^{2}\left(\Sigma_{\mu}\right)$ by

$$
(Q \xi)\left(s, \mu^{r}\right)= \begin{cases}\xi\left(s, \mu^{r}\right) & \text { if } s \text { is even } \\ 0 & \text { if } s \text { is odd }\end{cases}
$$

It is not hard to prove the following result.

Proposition 1.3. The operator $Q$ is a central projection in the multiplier algebra of $B$. One has

$$
\Phi(Q)=Q \otimes Q+(1-Q) \otimes(1-Q)
$$


Proof. Because $Q$ is a function of $N$, it is not hard to see that it is an element of the multiplier algebra $M(B)$. Because of the conditions on the joined spectrum of the pair $(N,|b|)$ it is also a function of $|b|$. By the normality of $b$ it follows that $Q$ also commutes with $u$. Therefore it is a central projection in $M(B)$. We can write $Q=\frac{1}{2}\left(1+(-1)^{N}\right)$ where $(-1)^{N}=f(N)$ with $f(k)=(-1)^{k}$ for $k \in \mathbb{Z}$. We have $\Phi\left((-1)^{N}\right)=(-1)^{N} \otimes(-1)^{N}$. Then the above formula for $\Phi(Q)$ follows from an easy calculation.

In fact, $Q$ is the spectral projection corresponding to the even part of the spectrum of $N$. Because of the relation between the spectrum of $N$ and of $|b|$, it is also a spectral projection of $|b|$. The projection $Q$ splits $B$ into a direct sum of parts where the spectra of $N$ and $|b|$ are no longer related. We will see later that these two parts of $B$ are isomorphic.

We now define some ${ }^{*}$-homomorphisms.

Lemma 1.4. There is $a^{*}$-homomorphism $\psi: B \rightarrow \mathbb{C}$ defined by

$$
\psi\left(\sum_{k} u^{k} f_{k}(N,|b|)\right)=f_{0}(1,0) .
$$

This is obtained by considering the trivial pair of operators 1 and 0 on the Hilbert space $\mathbb{C}$ and using the universal property of $B$. Remark that $\psi(Q)=0$.

Lemma 1.5. Define $\rho=(\psi \otimes \iota) \Phi$. This is the ${ }^{*}$-automorphism of $B$ corresponding to replacing $N$ by $N+1$ and $b$ by $\mu^{-\frac{1}{2}} b$.

Proof. Formally we have

$$
\begin{aligned}
\rho(N) & =(\psi \otimes \iota) \Phi(N) \\
& =(\psi \otimes \iota)(N \otimes 1+1 \otimes N) \\
& =1+N \\
\rho(b) & =(\psi \otimes \iota) \Phi(b) \\
& =(\psi \otimes \iota)\left(b \otimes \mu^{\frac{1}{2} N}+\mu^{-\frac{1}{2} N} \otimes b\right) \\
& =\mu^{-\frac{1}{2}} b .
\end{aligned}
$$

This formal calculation can of course be made precise by considering the right functions of these operators. 
Remark that $\rho(Q)=1-Q$ so that $\rho$ is a ${ }^{*}$-automorphism that gives an ismomorphism between the two parts of $B$.

Lemma 1.6. Define $\theta=(\psi \otimes \iota \otimes \psi)(\Phi \otimes \iota) \Phi$. This is the ${ }^{*}$-automorphism of $B$ corresponding to replacing $N$ by $N+2$ arıd by leaving b invariant.

Proof.

$$
\begin{aligned}
\theta(N) & =(\rho \otimes \psi) \Phi(N) \\
& =(\rho \otimes \psi)(N \otimes 1+1 \otimes N) \\
& =N+1+1=N+2 \\
\theta(b) & =(\rho \otimes \psi) \Phi(b) \\
& =(\rho \otimes \psi)\left(b \otimes \mu^{\frac{1}{2} N}+\mu^{-\frac{1}{2} N} \otimes b\right) \\
& =\mu^{-\frac{1}{2}} b \mu^{\frac{1}{2}}=b .
\end{aligned}
$$

Remark that $\theta(Q)=Q$.

We will also use the ${ }^{*}$-endomorphism $\gamma$ of $B$ obtained by leaving $N$ and replacing $b$ by 0 .

\section{Unitary representations of $\widehat{E}_{\mu}(2)$.}

A unitary representation of $\widehat{E}_{\mu}(2)$ on some Hilbert space $\mathcal{H}$ is a unitary element $W$ in the multiplier algebra $M(B \otimes C B(\mathcal{H}))$, where $C B(\mathcal{H})$ denotes the $\mathrm{C}^{*}$-algebra of compact operators on $\mathcal{H}$, satisfying the equation

$$
(\Phi \otimes \iota) W=W_{23} W_{13} .
$$

Note that this formula differs from the usual one but the difference is not essential. We use it in this form because we work with the dual and we want to obtain the same formula for the bicharacter as in [2].

We will prove the following result.

Theorem 2.1. If $W$ is as above, then there exist a unitary operator $v$ and a normal operator $n$ on $\mathcal{H}$ such that $\operatorname{Sp}(n) \subseteq \overline{\mathbb{C}}^{\mu}$ and $v n v^{*}=\mu n$ and such that

$$
W=F_{\mu}\left(\mu^{\frac{1}{2} N} b \otimes v n\right)(1 \otimes v)^{N \otimes 1} .
$$


Here $F_{\mu}$ is the function on normal operators with spectrum in $\overline{\mathbb{C}}^{\mu}$ as defined in $[\mathbf{3}]$. By $(1 \otimes v)^{N \otimes 1}$ we mean the operator $\sum_{k \in \mathbb{Z}} e_{k} \otimes v^{k}$ when $N=\sum_{k \in \mathbb{Z}} k e_{k}$ is the spectral resolution of $N$.

So, in what follows, fix a Hilbert space $\mathcal{H}$ and a unitary $W$ as above. We will first obtain the unitary $v$.

Definition 2.2. Define $v=(\psi \otimes \iota)(W)$.

This gives a unitary on $\mathcal{H}$. We will need the following formula.

Lemma 2.3. When $\gamma$ is the endomorphism of $B$ obtained by leaving $N$ invariant and sending $b$ to 0 , we get $(\gamma \otimes \iota)(W)=(1 \otimes v)^{N \otimes 1}$.

Proof. For any integer $s$ we consider the ${ }^{*}$-homomorphism $\psi_{s}$ from $B$ to $\mathbb{C}$ defined by the representation $(s, 0)$ of the pair $(N, b)$. Recall that $\psi=\psi_{1}$. Then, it is easy to see that $\psi_{s} \psi_{t}=\psi_{s+t}$ for all $s, t \in \mathbb{Z}$. So we find that $\left(\psi_{s} \otimes \iota\right)(W)=v^{s}$. Because $\gamma$ is induced by the pair $(N, 0)$, it is clear that $(\gamma \otimes \iota)(W)=(1 \otimes v)^{N \otimes 1}$.

Because $\rho=(\psi \otimes \iota) \Phi$ we get

$$
\begin{aligned}
(\rho \otimes \iota)(W) & =(\psi \otimes \iota \otimes \iota)(\Phi \otimes \iota) W \\
& =(\psi \otimes \iota \otimes \iota)\left(W_{23} W_{13}\right) \\
& =W(1 \otimes v) .
\end{aligned}
$$

And since $\rho(Q)=1-Q$ it follows that

$$
W((1-Q) \otimes 1)=(\rho \otimes \iota)(W(Q \otimes 1))\left(1 \otimes v^{*}\right) .
$$

So we see that it will be sufficient to determine the part $W(Q \otimes 1)$. Therefore, we will work now mainly in the subspace $Q \ell^{2}\left(\Sigma_{\mu}\right)$. We will denote restrictions of operators, ${ }^{*}$-automorphisms, ... to this subspace with an index 0 . We will use e.g. $W_{0}$ for $W(Q \otimes 1), \theta_{0}$ for the map $x Q \rightarrow \theta(x Q), \ldots$ We will use $\Phi_{0}$ for the map $x Q \rightarrow \Phi(x Q)(Q \otimes Q)$. Notice that $\operatorname{Sp} N_{0} \subseteq 2 \mathbb{Z}$ where $N_{0}=N Q$.

Lemma 2.4. Define $U_{0}=(1 \otimes v)^{-\frac{1}{2} N_{0} \otimes 1} W_{0}(1 \otimes v)^{-\frac{1}{2} N_{0} \otimes 1}$. Then $\left(\theta_{0} \otimes \iota\right)\left(U_{0}\right)=U_{0}$.

Proof. We have

$$
\begin{aligned}
(\theta \otimes \iota)(W) & =(\psi \otimes \iota \otimes \psi \otimes \iota)(\Phi \otimes \iota \otimes \iota)(\Phi \otimes \iota)(W) \\
& =(\psi \otimes \iota \otimes \psi \otimes \iota)\left(W_{34} W_{24} W_{14}\right) \\
& =(1 \otimes v) W(1 \otimes v) .
\end{aligned}
$$


And because $\theta(N)=N+2$ we get

$$
\begin{aligned}
\left(\theta_{0} \otimes \iota\right)\left(U_{0}\right) & =(1 \otimes v)^{-\frac{1}{2}\left(N_{0}+2\right) \otimes 1}(1 \otimes v) W_{0}(1 \otimes v)(1 \otimes v)^{-\frac{1}{2}\left(N_{0}+2\right) \otimes 1} \\
& =(1 \otimes v)^{-\frac{1}{2} N_{0} \otimes 1} W_{0}(1 \otimes v)^{-\frac{1}{2} N_{0} \otimes 1} \\
& =U_{0}
\end{aligned}
$$

Lemma 2.5. There is a continuous function $f: \operatorname{Sp}\left(b_{0}\right) \rightarrow \mathcal{B}(\mathcal{H})$ such that $f(z)$ is a unitary operator for all $z \in \operatorname{Sp}\left(b_{0}\right)$ and $U_{0}=f\left(b_{0}\right)$.

Proof. Let $B \otimes C B(\mathcal{H})$ act on $\ell^{2}\left(\Sigma_{\mu}\right) \otimes \mathcal{H}$. Then $W$ is a unitary operator on this space. Hence $W_{0}$ is a unitary operator on $Q \ell^{2}\left(\Sigma_{\mu}\right) \otimes \mathcal{H}$. Since the ${ }^{*}$-automorphism $\theta_{0}$ is implemented by the unitary $u_{0}$, defined by

$$
\left(u_{0} \xi\right)\left(s, \mu^{r}\right)=\xi\left(s-2, \mu^{r}\right)
$$

we must have that $U_{0}$ commutes with this operator $u_{0} \otimes 1$. Recall that $u_{0}\left|b_{0}\right|$ is the polar decomposition of $b_{0}$. Because $|b|$ commutes with all of $B$, also $U_{0}$ commutes with $\left|b_{0}\right| \otimes 1$. Hence $U_{0}$ commutes with all functions of $b_{0}$. But these generate a maximal abelian subalgebra $\mathcal{M}$ on $Q \ell^{2}\left(\Sigma_{\mu}\right)$. So $U_{0} \in \mathcal{M} \otimes \mathcal{B}(\mathcal{H})$, the von Neumann tensor product of $\mathcal{M}$ with $\mathcal{B}(\mathcal{H})$. This means that $U_{0}$ has the form $f\left(b_{0}\right)$ where $f: \operatorname{Sp}\left(b_{0}\right) \rightarrow \mathcal{B}(\mathcal{H})$ is a measurable function with values in the unitary operators. However, $U_{0}$ is in the multiplier algebra of $Q B \otimes C \mathcal{B}(\mathcal{H})$. And the fixed points in $Q B$ for the automorphism $\theta_{0}$ are clearly of the form $g\left(b_{0}\right)$ with $g \in C_{\infty}\left(\operatorname{Sp}\left(b_{0}\right)\right)$. Therefore, $f$ must be a continuous function.

Lemma 2.6. We have

$$
f(R \dot{+} S)=f(R) f(S)
$$

when $R=\mu^{-\frac{1}{2} N_{0}} \otimes b_{0}$ and $S=b_{0} \otimes \mu^{\frac{1}{2} N_{0}}$.

Proof. Consider again the formula

$$
(\Phi \otimes \iota) W=W_{23} W_{13}
$$

and cut down by $Q \otimes Q \otimes 1$. Recall that $W_{0}=(1 \otimes v)^{\frac{1}{2} N_{0} \otimes 1} f\left(b_{0}\right)(1 \otimes v)^{\frac{1}{2} N_{0} \otimes 1}$ So we obtain for the left hand side :

$(1 \otimes 1 \otimes v)^{\frac{1}{2}\left(N_{0} \otimes 1 \otimes 1+1 \otimes N_{0} \otimes 1\right)}$

$$
\cdot f\left(b_{0} \otimes \mu^{\frac{1}{2} N_{0}}+\mu^{-\frac{1}{2} N_{0}} \otimes b_{0}\right)(1 \otimes 1 \otimes v)^{\frac{1}{2}\left(N_{0} \otimes 1 \otimes 1+1 \otimes N_{0} \otimes 1\right)}
$$


Now consider the endomorphism $\gamma$ that takes $N$ to $N$ and $b$ to 0 . We showed in Lemma 2.3 that $(\gamma \otimes \iota)(W)=(1 \otimes v)^{N \otimes 1}$. If we apply this to the first factor in the tensor product we obtain

$(1 \otimes 1 \otimes v)^{\frac{1}{2}\left(N_{0} \otimes 1 \otimes 1+1 \otimes N_{0} \otimes 1\right)} f\left(\mu^{-\frac{1}{2} N_{0}} \otimes b_{0}\right)(1 \otimes 1 \otimes v)^{\frac{1}{2}\left(N_{0} \otimes 1 \otimes 1+1 \otimes N_{0} \otimes 1\right)}$

$$
=\left(W_{0}\right)_{23}(1 \otimes 1 \otimes v)^{N_{0} \otimes 1 \otimes 1} .
$$

If we apply it to the second factor we get

$(1 \otimes 1 \otimes v)^{\frac{1}{2}\left(N_{0} \otimes 1 \otimes 1+1 \otimes N_{0} \otimes 1\right)} f\left(b_{0} \otimes \mu^{\frac{1}{2} N_{0}}\right)(1 \otimes 1 \otimes v)^{\frac{1}{2}\left(N_{0} \otimes 1 \otimes 1+1 \otimes N_{0} \otimes 1\right)}$

$$
=(1 \otimes 1 \otimes v)^{1 \otimes N_{0} \otimes 1}\left(W_{0}\right)_{13} .
$$

If we multiply these two formulas we get

$$
\begin{aligned}
\left(W_{0}\right)_{23}\left(W_{0}\right)_{13}= & (1 \otimes 1 \otimes v)^{\frac{1}{2}\left(N_{0} \otimes 1 \otimes 1+1 \otimes N_{0} \otimes 1\right)} f\left(\mu^{-\frac{1}{2} N_{0}} \otimes b_{0}\right) \\
& (1 \otimes 1 \otimes v)^{\frac{1}{2}\left(N_{0} \otimes 1 \otimes 1+1 \otimes N_{0} \otimes 1\right)-\left(N_{0} \otimes 1 \otimes 1\right)} \\
& (1 \otimes 1 \otimes v)^{-\left(1 \otimes N_{0} \otimes 1\right) \dot{1} \frac{1}{2}\left(N_{0} \otimes 1 \otimes 1+1 \otimes N_{0} \otimes 1\right)} \\
& f\left(b_{0} \otimes \mu^{\frac{1}{2} N_{0}}\right)(1 \otimes 1 \otimes v)^{\frac{1}{2}\left(N_{0} \otimes 1 \otimes 1+1 \otimes N_{0} \otimes 1\right)} .
\end{aligned}
$$

If we combine this with the formula in the beginning, we precisely get the desired result.

These operators $R$ and $S$ precisely satisfy the conditions of the pairs considered in $[\mathbf{3}]$. Indeed

$$
\begin{aligned}
& R=\mu^{-\frac{1}{2} N_{0}} \otimes b_{0}=\left(1 \otimes u_{0}\right)\left(\mu^{-\frac{1}{2} N_{0}} \otimes\left|b_{0}\right|\right) \\
& S=b_{0} \otimes \mu^{\frac{1}{2} N_{0}}=\left(u_{0} \otimes 1\right)\left(\left|b_{0}\right| \otimes \mu^{\frac{1}{2} N_{0}}\right)
\end{aligned}
$$

and $\left|b_{0}\right|$ and $N_{0}$ strongly commute, $\operatorname{Sp} N_{0}=2 \mathbb{Z}, \operatorname{Sp}\left|b_{0}\right|=\overline{\mathbb{C}}_{\mu}, u_{0}^{*} N_{0} u_{0}=$ $N_{0}+2 Q$. Therefore we can apply Theorem 4.2 of [3].

Lemma 2.7. There exist a normal operator $n$ on $\mathcal{H}$ with $\operatorname{Sp}(n) \subseteq \overline{\mathbb{C}}^{\mu}$ such that

$$
f\left(b_{0}\right)=F_{\mu}\left(b_{0} \otimes n\right)
$$

where $F_{\mu}$ is as in [2].

Lemma 2.8. We have $v n v^{*}=\mu n$.

Proof. Apply the ${ }^{*}$-automorphism $\rho^{2}$ to the formula

$$
W_{0}=(1 \otimes v)^{\frac{1}{2}\left(N_{0} \otimes 1\right)} F_{\mu}\left(b_{0} \otimes n\right)(1 \otimes v)^{\frac{1}{2}\left(N_{0} \otimes 1\right)} .
$$


We know that $\rho^{2}\left(N_{0}\right)=N_{0}+2, \rho^{2}\left(b_{0}\right)=\mu^{-1} b_{0}$ and $\rho^{2}\left(W_{0}\right)=W_{0}\left(1 \otimes v^{2}\right)$. So we get

$$
\begin{aligned}
W_{0}\left(1 \otimes v^{2}\right) & =(1 \otimes v)^{\frac{1}{2}\left(N_{0} \otimes 1\right)}(1 \otimes v) F_{\mu}\left(\mu^{-1} b_{0} \otimes n\right)(1 \otimes v)(1 \otimes v)^{\frac{1}{2}\left(N_{0} \otimes 1\right)} \\
W_{0} & =(1 \otimes v)^{\frac{1}{2}\left(N_{0} \otimes 1\right)} F_{\mu}\left(\mu^{-1} b_{0} \otimes v n v^{*}\right)(1 \otimes v)^{\frac{1}{2}\left(N_{0} \otimes 1\right)} .
\end{aligned}
$$

By uniqueness we get $v n v^{*}=\mu n$.

Lemma 2.9. We have $W_{0}=F_{\mu}\left(\mu^{\frac{1}{2} N_{0}} b_{0} \otimes n v\right)(1 \otimes v)^{N_{0} \otimes 1}$.

Proof.

$$
\begin{aligned}
(1 \otimes v)^{\frac{1}{2}\left(N_{0} \otimes 1\right)} & \left(b_{0} \otimes n\right)(1 \otimes v)^{-\frac{1}{2}\left(N_{0} \otimes 1\right)} \\
& =(1 \otimes v)^{\frac{1}{2}\left(N_{0} \otimes 1\right)}\left(u_{0}\left|b_{0}\right| \otimes n\right)(1 \otimes v)^{-\frac{1}{2}\left(N_{0} \otimes 1\right)} \\
& =\left(u_{0} \otimes 1\right)(1 \otimes v)^{\frac{1}{2}\left(N_{0} \otimes 1\right)}(1 \otimes v)\left(\left|b_{0}\right| \otimes n\right)(1 \otimes v)^{-\frac{1}{2}\left(N_{0} \otimes 1\right)} \\
& =\left(u_{0} \otimes 1\right)\left(\mu^{\frac{1}{2} N_{0}}\left|b_{0}\right| \otimes v n\right) \\
& =\mu^{\frac{1}{2} N_{0}} b_{0} \otimes \mu^{-1} v n
\end{aligned}
$$

From this we see that

$$
W(Q \otimes 1)=F_{\mu}\left(\mu^{\frac{1}{2} N} b Q \otimes n v\right)(1 \otimes v)^{N \otimes 1}(Q \otimes 1) .
$$

If we apply $\rho$ we get

$$
\begin{aligned}
& W(1 \otimes v)((1-Q) \otimes 1) \\
& \quad=F_{\mu}\left(\mu^{\frac{1}{2}(N+1)} \mu^{-\frac{1}{2}} b(1-Q) \otimes n v\right)(1 \otimes v)^{(N+1) \otimes 1}(1-Q) \otimes 1
\end{aligned}
$$

and so

$$
W((1-Q) \otimes 1)=F_{\mu}\left(\mu^{\frac{1}{2} N} b(1-Q) \otimes n v\right)(1 \otimes v)^{N \otimes 1}(1-Q) \otimes 1 .
$$

Therefore we get precisely the same formula on $Q$ and $(1-Q)$. Hence, if we replace $n$ by $v n v^{*}=\mu n$, we get the formula in our theorem.

\section{References}

[1] S.L. Woronowicz, Unbounded elements affiliated with $C^{*}$-algebras and non-compact quantum groups, Commun. Math. Phys., 136 (1991), 399-432.

[2] , Quantum E(2) group and its Pontryagin dual, Lett. in Math. Phys., 23 (1991), 251-263. 
[3] Operator equalities related to the quantum E(2) group, Commun. Math. Phys., 144 (1992), 417-428.

[4] S.L. Woronowicz and K. Napiórkowski, Operator theory in the $C^{*}$-algebra framework, Rep. Math. Phys., 31 (1992), 353-373.

[5] A. Van Daele, Dual Pairs of Hopf ${ }^{*}$-algebras, Lecture notes (first version). K.U. Leuven, (1991).

[6] Dual Pairs of Hopf * -algebras, Lecture notes (second version). Bull. of the Lond. Math. Soc., 25 (1993), 209-230.

[7] The operator $a \otimes b+b \otimes a^{-1}$ when $a b=\lambda b a$, Preprint K.U. Leuven, (1989).

[8] H.T. Koelink, On quantum groups and q-special functions, Ph.D. Thesis Leiden, (1991).

[9] A. Van Daele, A quantum deformation of the Heisenberg group, Preprint K.U. Leuven, (1989).

Received October 6, 1993 and revised June 1, 1994. The second author was supported by Komitet Badan Naukowych, grant No 21129101.

KATHOLEIKE UNIVERSITEIT LEUVEN

Celestijnenlaan 200B

3001 LEUVEN (HEVERLEE)

BELGIUM

E-mail address: alfons.vandaele@wis.kuleuven.ac.be 

Peng Lin and Richard Rochberg, Trace ideal criteria for Toeplitz and Hankel operators on the weighted Bergman spaces with exponential type

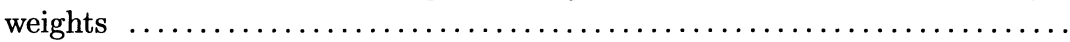

Donald E. Marshall and Arne Stray, Interpolating Blaschke products . .

Kathy D. Merrill and Lynne $\mathbf{H}$. Walling, On quadratic reciprocity over

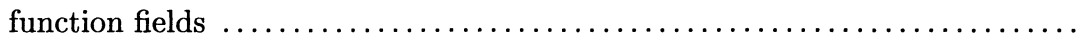

Takahiko Nakazi and Masahiro Yamada, $\left(A_{2}\right)$-conditions and Carleson

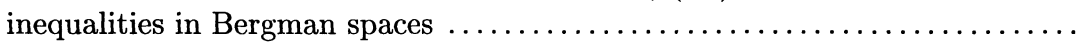

C. Ott, A note on a paper of E. Boasso and A. Larotonda ..............

Victor Patrangenaru, Classifying 3 and 4 dimensional homogeneous Riemannian manifolds by Cartan triples

Carlo Pensavalle and Tim Steger, Tensor products with anisotropic principal series representations of free groups

Ying Shen, On Ricci deformation of a Riemannian metric on manifold with boundary

Albert Jeu-Liang Sheu, The Weyl quantization of Poisson $S U(2) \ldots \ldots$

Alexandra Shlapentokh, Polynomials with a given discriminant over fields of algebraic functions of positive characteristic

Eric Stade and D.I. Wallace, Weyl's law for $S L(3, \mathbb{Z}) \backslash S L(3, \mathbb{R}) / S O(3, \mathbb{R})$

Christopher W. Stark, Resolutions modeled on ternary trees ........ 557

Per Tomter, Minimal hyperspheres in two-point homogeneous spaces .....

Jun Tomiyama, Topological Full groups and structure of normalizers in

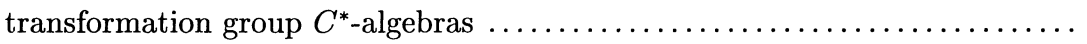

Nik Weaver, Subalgebras of little Lipschitz algebras 


\section{PACIFIC JOURNAL OF MATHEMATICS}

\section{Volume $173 \quad$ No. $2 \quad$ April 1996}

A mean value inequality with applications to Bergman space operators

PATRICK ROBERT AHERn and ZELJKO CUCKOVIC

$H^{p}$-estimates of holomorphic division formulas

MATS ANDERSSON and HASSE CARLSSON

Group structure and maximal division for cubic recursions with a double root

Christian JeAn-Claude Ballot

The Weil representation and Gauss sums

ANTONiA WiLson BLUHER

Duality for the quantum $E(2)$ group

ALFONS VAN DAELE and S. L. WORONOWICZ

Cohomology complex projective space with degree one codimension-two fixed submanifolds 387

KARL HEINZ DOVERMANN and ROBERT D. LITTLE

On the mapping intersection problem

ALEXANDER DRANISHNIKOV

From the $L^{1}$ norms of the complex heat kernels to a Hörmander multiplier theorem for

sub-Laplacians on nilpotent Lie groups

\section{XUAN THINH DUONG}

Isoperimetric inequalities for automorphism groups of free groups

Allen E. Hatcher and Karen Vogtmann

Approximation by normal elements with finite spectra in $C^{*}$-algebras of real rank zero

HUAXIN LIN

Interpolating Blaschke products

DonALD EdDY MARShall and ARNE STRAY

Interpolating Blaschke products generate $H^{\infty}$

JOHN BRADY GARNETT and ARTUR NICOLAU

Classifying 3- and 4-dimensional homogeneous Riemannian manifolds by Cartan triples

VICTOR PATRANGENARU

Polynomials with a given discriminant over fields of algebraic functions of positive

characteristic

ALEXANDRA SHLAPENTOKH

Resolutions modeled on ternary trees

CHRISTOPHER W. STARK

Topological full groups and structure of normalizers in transformation group $C^{*}$-algebras 